\title{
A gelatinous theory of everything
}

\author{
Cells, Gels and the Engines of Life: A New, Unifying Approach to \\ Cell Function \\ By Gerald H. Pollack \\ Ebner \& Sons, \$55.00, ISBN 0-9626895-1-3, 2001
}

Review ed by Robert $\mathbf{H}$. Insall

School of Biosciences, University of Birmingham, Birmingham, B15 2TT, UK

If Cells, Gels and the Engines of Life is correct, a large number of biologists should be fighting very scared. According to the author, Gerald Pollack, one of the fundamental concepts of modern cell biology is seriously wrong. Pollack believes that the importance of the plasma membrane has been overwhelmingly overstated, and pumps, transporters, channels, membranes bounding intracellular compartments and suchlike have few or none of the roles that we all ascribe to them. If this is all correct, a tidy proportion of all modern biologists have been wasting their time for the past few years. Plainly the many thousands of neurobiologists who base their work on neuronal ion channels will be troubled if, as

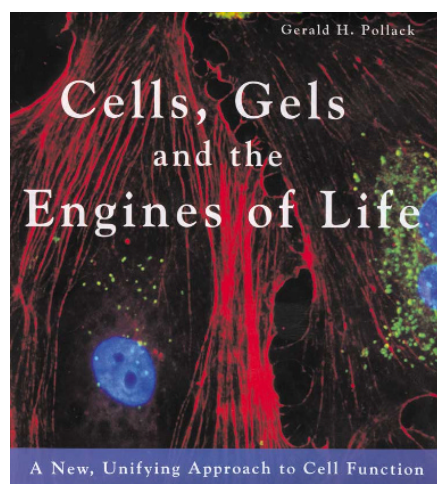

Pollack's view of the cell is fundamentally different. He sees cytoplasm as a selfrestricting architectural gel, potential gradients and ion gradients generated by specific inclusion or exclusion of separate ions, and no need for a boundary to separate compartments. Thus, when the plasma membrane is damaged (whether by electroporation, detergents, or other insults), the cell not only maintains its structure, but also its electrical potential and the separation of its subcompartments. Pumps and channels are simply pointless, and data from techniques such as patch-clamping are riddled with artifacts. The membranes around subcompartments such as exocytic vesicles are essentially irrelevant to their behavior, which Pollack more or less states, these channels are artifacts needed to shore up the old membrane-centered paradigm.

Pollack's central hypothesis is that the contents of cells are not aqueous solutions, as biochemists have tended to assume. Rather, the cytoplasm is a complex gel, and much of the behavior of cells can be explained by gel-specific concepts such as phase transitions and exclusion of specific solutes from the gel matrix. Historically, biologists invoked membrane-based barriers because they thought an impermeable bag was needed to hold the cell's innards in and keep the outside world out. This, for Pollack, is the fundamental error-a gel-like cytoplasm holds its own form without needing a bag to hold it. One mistake leads to another, however, and once people were erroneously committed to impermeable membranes, they had to invent channels to explain the passage of ions into the cell, and then pumps to explain why potential and ion gradients survived the action of the channels. In other words, much of membrane biology is a house of cards, built on a flawed foundation to "save the phenomena". be done to improve this. A theory of everything has to be shown to explain quite a lot of things, after all. Darwin's Origin of Species, which I read while I was still a keen student, contains long stretches that were insufferable to read but important for the argument. It also didn't help that the book arrived during a British general election, so the airwaves were crammed with politicians authoritatatively explaining why the opposition was obviously flawed and everything should be changed. I suspect all that hot air induced a backwash of cynicism about new ideas and demands for change.

This is the book of someone who knows the implications of what he's trying to say. Chapters and sections have titles like "Debunking Myths," "Toward Ground Truth," "Building from Basics" and "A New Paradigm for Cell Function". The critiques of existing models are strongly argued. There's no room for the current view to be basically sound, give or take a reinterpretation here and there, or revision of a small subfield. The only answer is a complete paradigm shift, with the old membrane-based framework going out of the window and being completely replaced by a complex and interlocking system of phase transitions in an architecture built from gels.

And here, for me, lies the problem. Paradigm shifts are supposed to arise when the old framework of knowledge starts to creak so badly that scientists in the field can see the gaps. Newer paradigms arise because they are better at explaining the difficult results which trip up the older ones. From where I stand, however, the membrane story is doing pretty well. The structure of the mitochondrial ATPase has been solved, and fits very nicely with the concept of energy generated from proton gradients across membranes. Lipid-soluble drugs that insert in the membrane and dissipate charge gradients have the expected results on energy generation. The long and growing list of drugs that bind to the maligned neural ion channels have, by and large, the expected effects on cell behavior, and several of them work in the expected way to change nerve impulses and cure diseases. There are many such points-I don't have space to raise them all, and I'm sure the author could raise a counterargument for each one. Overall, suffice it to say that I was intrigued, and that I found elements of the argument completely convincing. I was not, however, convinced that membranes are overvalued and the existing paradigm is tottering. If you feel like taking on a big argument, why not see if you agree? 